\title{
Statistical machine translation of subtitles: From OpenSubtitles to TED
}

Müller, Mathias ; Volk, Martin

\begin{abstract}
In this paper, we describe how the differences between subtitle corpora, OpenSubtitles and TED, influence machine translation quality. In particular, we investigate whether statistical machine translation systems built on their basis can be used interchangeably. Our results show that OpenSubtiles and TED contain very different kinds of subtitles that warrant a subclassification of the genre. In addition, we have taken a closer look at the translation of questions as a sentence type with special word order. Interestingly, we found the BLEU scores for questions to be higher than for random sentences.
\end{abstract}

DOI: https://doi.org/10.1007/978-3-642-40722-2_14

Posted at the Zurich Open Repository and Archive, University of Zurich

ZORA URL: https://doi.org/10.5167/uzh-82233

Book Section

Accepted Version

Originally published at:

Müller, Mathias; Volk, Martin (2013). Statistical machine translation of subtitles: From OpenSubtitles to TED. In: Gurevych, Iryna; Biemann, Chris; Zesch, Torsten. Language Processing and Knowledge in the Web. Berlin Heidelberg: Springer, 132-138.

DOI: https://doi.org/10.1007/978-3-642-40722-2_14 


\title{
Statistical Machine Translation of Subtitles: From OpenSubtitles to TED
}

\author{
Mathias Müller and Martin Volk \\ Institute of Computational Linguistics, Zurich, Switzerland
}

\begin{abstract}
In this paper, we describe how the differences between subtitle corpora, OpenSubtitles and TED, influence machine translation quality. In particular, we investigate whether statistical machine translation systems built on their basis can be used interchangeably. Our results show that OpenSubtiles and TED contain very different kinds of subtitles that warrant a subclassification of the genre. In addition, we have taken a closer look at the translation of questions as a sentence type with special word order. Interestingly, we found the BLEU scores for questions to be higher than for random sentences.
\end{abstract}

\section{Introduction}

The key ingredient for building successful Statistical Machine Translation (SMT) systems is a suitable and sufficiently large parallel corpus. For a number of language pairs, large subtitle corpora are available. The OPUS OpenSubtitles corpus (Tiedemann, 2009) contains fansubs for 54 languages. This collection amounts to several million parallel sentences for the most popular language pairs. On the other hand, there is the collection of subtitles from the TED talks (Cettolo et al., 2012), a series of high quality talks on "Technology, Entertainment, and Design". Although the TED collection is much smaller, it is interesting because of its wide coverage and complexity of topics.

Therefore, we set out to investigate the degree of similarity between the subtitles in the two corpora and to what extent this influences the quality of SMT systems trained on them. As a side issue we wanted to check the general usefulness of the OpenSubtitles corpus for training SMT systems which has been questioned repeatedly in the literature (see e.g. (Petukhova et al., 2012)). In order to gain deeper insight into the impact of the two corpora on specific linguistic phenomena, we evaluated their translation quality on questions.

In this paper we describe the differences between the two subtitle corpora and their impact on translation quality. We trained a number of Moses systems for that purpose, using parallel subtitles in English, French and German. In all, we trained twelve systems under the exact same conditions (preprocessing, Moses command line options). Each system was then tested on three test sets including a set containing only questions. 


\section{OpenSubtitles vs. TED}

Both OpenSubtitles and TED are collections of parallel sentences derived from subtitles. So, we are dealing with sentence-aligned corpora and not subtitlealigned corpora as, for instance, Volk et al. (2010). Contrary to our intuition, Volk et al. (2010) found that subtitle-aligned corpora are as good for building SMT systems for subtitles as sentence-aligned corpora. The average sentence length is around 6 words per sentence for OpenSubtitles and around 17 for TED, irrespective of the language. It is surprising that the figures across languages are so similar. We believe that this is an artifact of the automatic alignment. Only those sentences that are of similar length were aligned. However, these numbers clearly indicate that OpenSubtitles and TED subtitles are different from one another. Some randomly chosen lines may illustrate this point.

\section{Example 1. Subtitle examples from OpenSubtitles and TED ${ }^{1}$}

OpenSubs: You miss me, today?

OpenSubs: Faut y penser avant.

TED: The first law is two-colorability: You can color any crease pattern with just two colors without ever having the same color meeting.

TED: Et avec ce qu'il trouve sur place, il entre et fait son petit studio qui sert de base de travail.

The differences in length can be explained if we consider the circumstances: OpenSubtitles are taken from regular movies and series, where sentences tend to be short and where subtitles are shortened to fit on the screen in the available time. TED talks on the other hand treat rather complex subjects that in turn demand more complex sentences. Any differences may have an influence on MT performance when crosstesting between corpora, more precisely on BLEU scores in our case. For our experiments, we used OpenSubtitles, as prepared in the OPUS project, for language pairs between EN, FR and DE in all possible combinations. The OpenSubtitles collections for these languages are large, ranging from 2.8 million sentence pairs for DE-FR up to 20 million sentence pairs for ENFR. These corpora should be sufficient, given that Hardmeier and Volk (2009) argue that 1 million sentence pairs is suitable for subtitle SMT. Still we need to remember that subtitles in OpenSubtitles are of an unknown quality. Some are controlled and consistent, but others contain spelling errors or strange wordings. All texts are already sentence-aligned and formatted in a Moses-friendly way. As for the limitations of sentence-alignment techniques, see Tiedemann (2009).

The TED collection (Cettolo et al., 2012) is smaller. These subtitles are crawled from www.ted.com, a platform offering talks that were recorded at TED conferences or similar events. These videos sometimes come with subtitles translated into 30 or more languages by volunteers from within the TED community. The translations are generally high-quality because TED requires translators

\footnotetext{
${ }^{1}$ Obviously unrelated sentences, not translations that correspond.
} 
to peer-review their work and prove their proficiency. These corpora, too, have been preprocessed and sentence-aligned by (Cettolo et al., 2012) as to allow using them in Moses with ease. Again, we only used the parts in EN, FR and DE.

In total, we built 12 SMT systems between EN, FR and DE on the basis of 6 different corpora from TED and OpenSubtitles. We used the same material for both directions, for example the same corpus to translate EN-FR and FR-EN. Table 1 shows the corpora's sizes as the number of lines (roughly equal to the number of sentences) and the number of words using a naive tokenization.

\begin{tabular}{|c|c|c|c|}
\hline Corpus & Language & Number of lines & Number of words \\
\hline \multirow{2}{*}{ OpenSubtitles EN-DE (DE-EN) } & EN & $4,654,635$ & $26,266,191$ \\
& DE & $"$ & $27,189,072$ \\
\hline \multirow{2}{*}{ OpenSubtitles EN-FR (FR-EN) } & EN & $19,858,798$ & $119,682,551$ \\
& FR & $"$ & $115,456,439$ \\
\hline \multirow{2}{*}{ OpenSubtitles DE-FR (FR-DE) } & DE & $2,862,370$ & $16,946,049$ \\
& FR & $"$ & $16,818,332$ \\
\hline \hline \multirow{2}{*}{ TED EN-DE (DE-EN) } & EN & 63,865 & $1,029,090$ \\
& DE & $"$ & $1,034,657$ \\
\hline \multirow{2}{*}{ TED EN-FR (FR-EN) } & EN & 114,582 & $1,916,788$ \\
& FR &, & $2,000,958$ \\
\hline \multirow{2}{*}{ TED DE-FR (FR-DE) } & DE & 62,148 & 967,935 \\
& FR & $"$ & $1,056,758$ \\
\hline
\end{tabular}

Table 1. Corpus sizes

\section{Building SMT Systems}

Starting out with the corpora described in the section above, we built phrasebased Moses systems (Koehn et al., 2007) and tested their performance. Moses is used widely and is the state-of-the-art tool for statistical machine translation. We divided each corpus into training set (97 percent), tuning set (1 percent) and test set (2 percent) and assigned parallel lines randomly to the sets ${ }^{2}$. All of the data passed through the usual stages of preprocessing as we cleaned, lowercased and tokenized it using scripts offered by the Moses toolkit. For word-alignment we used GIZA++ (Och and Ney, 2003) which is implemented as part of Moses.

In order to build the language model we employed SRILM (Stolcke et al., 2011) together with Kneser-Ney discounting for smoothing, and interpolation as a back-off model for probabilities. These two options are an official recommendation by the Moses developers ${ }^{3}$. In general, we used the standard methods and

\footnotetext{
${ }^{2}$ While creating the test set via randomly allocating the lines is statistically sound, it might be more natural to test the subtitles of whole movies or series.

${ }^{3}$ See http://www.statmt.org/moses/?n=FactoredTraining/BuildingLanguageModel for further information.
} 
options where possible and consistently applied the same rules to all systems. The baseline systems were then tuned with MERT (also part of Moses), optimizing them with respect to the tuning set. After assembling complete Moses systems, we tested each on three different test sets to obtain BLEU scores.

\section{First Results}

We conducted several experiments on the TED and OpenSubtitles collections. In all cases, the performance of the MT systems was measured with multibleu, a script distributed with Moses. Table 2 reports the BLEU scores of our systems. The most straightforward test set for each system is the 2 percent of the original corpus set aside at the beginning, the "native test set". In contrast, the "foreign test set" is the native set's equivalent from the other collection. In other words, TED systems are subjected to foreign test data taken from OpenSubtitles and vice versa.

\begin{tabular}{|c|c|c|c|}
\hline Language pair & System & \multicolumn{2}{|c|}{ Test set } \\
\cline { 3 - 4 } & & native & foreign \\
\hline \multirow{2}{*}{ DE-EN } & OpenSubtitles & 27.92 & 20.56 \\
& TED & 25.06 & 14.29 \\
\hline \multirow{2}{*}{ DE-FR } & OpenSubtitles & 17.18 & 14.65 \\
& TED & 17.64 & 9.69 \\
\hline \multirow{2}{*}{ EN-DE } & OpenSubtitles & 19.55 & 16.93 \\
& TED & 24.38 & 12.59 \\
\hline \multirow{2}{*}{ EN-FR } & OpenSubtitles & 22.86 & 23.56 \\
& TED & 31.87 & 14.89 \\
\hline \multirow{2}{*}{ FR-DE } & OpenSubtitles & 13.42 & 10.72 \\
& TED & 13.12 & 8.34 \\
\hline \multirow{2}{*}{ FR-EN } & OpenSubtitles & 23.52 & $\mathbf{2 4 . 9 2}$ \\
& TED & $\mathbf{3 3 . 3 7}$ & 16.87 \\
\hline
\end{tabular}

Table 2. Performance results in BLEU scores

First, let us consider the difference in performance between OpenSubtitles and TED systems when confronted with their native test sets. Out of six OpenSubtitles systems, the highest scores are achieved with DE-EN, scoring almost 28. Only TED systems surpass 30, and only when translating between EN and FR. Thus, good performance results wherever EN is involved, irrespective of its being the source or target language. On the other hand, combinations of DE and FR lead to the lowest scores. To understand this, we have to bear in mind that most movies and all TED talks are in English in the first place. Often, the English transcription is done first and translators base their work on English subtitles. Therefore, combinations with English (EN-FR, FR-EN, DE-EN, EN-DE) can be expected to be translations of one another, whereas combinations between DE and FR (DE-FR, FR-DE) are not directly related. 
With regard to the foreign sets, the performance ranking changes somewhat. OpenSubtitles translating from FR to EN now takes the lead, resulting in a BLEU score of approximately 25. In general, the TED systems are affected more severely when confronted with foreign test lines, their scores plummeting to 50 percent of the former value in some cases. This indicates that the OpenSubtitles systems are more apt at translating TED than the other way round. Also, it implies that TED systems are more overfitted and OpenSubtitles systems more universal if our goal is to translate subtitles in general.

\section{Investigating MT Quality for Questions}

As a case study we have investigated the MT quality of questions. Questions are special because they have word order that is different from assertive clauses, and they use question words and special auxiliary verbs. For the sake of simplicity, a question is a line ending with a question mark. Here are some typical questions.

Example 2. Question examples from OpenSubtitles and TED

OpenSubs: Is the needle in his femoral artery, Mr. Palmer?

OpenSubs: Für dich sind wir nur Leichen, oder?

TED: And we asked ourselves, why couldn't it be exhibitionistic, like the Met, or like some of the other buildings at Lincoln Center?

TED: Was ist die Botschaft, was ist das Vokabular und die Grammatik, die von diesem Gebäude ausgesandt wird, und was sagt es uns über uns selbst?

A line that ends in a question mark in some cases might not be a question. For example, the English TED sentence in the example above is an assertion or an indirect question. But such cases are rare and are ignored here. Some of the lines ending with a question mark in one language do not have an equivalent counterpart, i.e. in the translation there is no question mark at the end. We disregarded them for our tests.

Our questions test set contains only questions from the native test set. We used the "question set" both for a quantitative (performance measures in BLEU scores) and qualitative analysis (manual error categorisation). With respect to questions, OpenSubtitles systems performed slightly better compared to the native test set, their scores climbing one or two BLEU points (see table 3). The scores of TED systems adapted slightly. Given the differences in performance between the native and question set, questions surprisingly score higher than the average subtitle of any type. We speculate that this might be due to the fact that questions are shorter than the average subtitle. The latter is 33.7 characters long - calculated over all the lines we took from OpenSubtitles. The average question line taken from OpenSubtitles counts no more than 27.2 characters. We get similar values for the TED corpora.

In order to evaluate the performance of translating questions qualitatively, we have looked at up to 100 translated questions from EN-DE and FR-DE, both 


\begin{tabular}{|c|c|c|}
\hline \multirow{2}{*}{ System } & \multicolumn{2}{|c|}{ Test set } \\
\cline { 2 - 3 } & native & questions \\
\hline OpenSubtitles DE-EN & 27.92 & 31.11 \\
\hline TED DE-EN & 25.06 & 27.09 \\
\hline OpenSubtitles DE-FR & 17.18 & 19.61 \\
\hline TED DE-FR & 17.64 & 17.14 \\
\hline OpenSubtitles EN-DE & 19.55 & 21.73 \\
\hline TED EN-DE & 24.38 & 27.99 \\
\hline OpenSubtitles EN-FR & 22.86 & 23.28 \\
\hline TED EN-FR & 31.87 & 29.7 \\
\hline OpenSubtitles FR-DE & 13.42 & 15.37 \\
\hline TED FR-DE & 13.12 & 15.37 \\
\hline OpenSubtitles FR-EN & 23.52 & 23.55 \\
\hline TED FR-EN & 33.37 & 30.61 \\
\hline
\end{tabular}

Table 3. BLEU scores for native and questions test sets

OpenSubs and TED. In particular, we have paid attention to the types of errors that occur. Our categories are fragmentation (translation unit span too narrow), omission, lack of agreement, difficulty with ambiguous terms, reordering, ulexis issues and addition (of a phrase). The following errors were repeatedly made. Systems translating from FR to DE frequently omitted an infinitive, whereas this never happened when translating from EN to DE. Also, only EN-DE systems treated many auxiliary verbs as full verbs. Out-of-vocabulary problems are a more serious issue with FR-DE, presumably because verb-pronoun compounds like "atterrissez-vous" or "a-t-il" are common in French. The majority of lexis errors is concerned with a hyphenated French word like those. Deliberately tokenizing these forms as part of the preprocessing would alleviate this effect.

\section{Conclusion}

Parallel corpora of subtitles are a valuable source for machine translation and are frequently used. We compared corpora from the TED and OpenSubtitles collections, and we suggest that "subtitles" is in fact too broad a category. Four rows of test sets revealed that the systems can hardly be used interchangeably, since sentence length, broad applicability and subtitle quality mark stark differences between subtitles from OpenSubtitles and from TED. They may be so different that they might best be treated as different genres indeed. We isolated questions and found slightly better BLEU scores for them as compared to randomly selected sentences.

In future studies, it might prove fruitful to incorporate data from both collections into one system and assign weights to each in order to counteract the different sizes of the training corpora. One way to achieve this is to combine the phrase tables resulting from building translation models (see Sennrich (2012)). 


\section{Bibliography}

Cettolo, M., Girardi, C., and Federico, M. (2012). Wit ${ }^{3}$ : Web inventory of transcribed and translated talks. In Proceedings of the $16^{\text {th }}$ Conference of the European Association for Machine Translation (EAMT), pages 261-268, Trento, Italy.

Hardmeier, C. and Volk, M. (2009). Using linguistic annotations in statistical machine translation of film subtitles. In Nodalida.

Koehn, P., Hoang, H., Birch, A., Callison-Burch, C., Federico, M., Bertoldi, N., Cowan, B., Shen, W., Moran, C., Zens, R., Dyer, C., Bojar, O., Constantin, A., and Herbst, E. (2007). Moses: Open source toolkit for statistical machine translation. In $A C L$.

Och, F. J. and Ney, H. (2003). A systematic comparison of various statistical alignment models. Computational Linguistics, 29(1):19-51.

Petukhova, V., Agerri, R., Fishel, M., Penkale, S., del Pozo, A., Maucec, M. S., Way, A., Georgakopoulou, P., and Volk, M. (2012). SUMAT: Data collection and parallel corpus compilation for machine translation of subtitles. In $L R E C$, pages $21-28$.

Sennrich, R. (2012). Perplexity minimization for translation model domain adaptation in statistical machine translation. In Proceedings of the 13th Conference of the European Chapter of the Association for Computational Linguistics (EACL), Avignon, France.

Stolcke, A., Zheng, J., Wang, W., and Abrash, V. (2011). SRILM at sixteen: Update and outlook. In Proceedings IEEE Automatic Speech Recognition and Understanding Workshop.

Tiedemann, J. (2009). News from opus - a collection of multilingual parallel corpora with tools and interfaces. In Nicolov, N., Bontcheva, K., Angelova, G., and Mitkov, R., editors, Recent Advances in Natural Language Processing, volume V, pages 237-248. John Benjamins, Amsterdam/Philadelphia, Borovets, Bulgaria.

Volk, M., Sennrich, R., Hardmeier, C., and Tidström, F. (2010). Machine translation of TV subtitles for large scale production. In Proceedings of the Second Joint EM+/CNGL Workshop on "Bringing MT to the User: Research on Integrating MT in the Translation Industry", pages 53-62, Denver. 